\title{
Dielectrophoretic Trapping of Dissociated Fetal Cortical Rat Neurons
}

\author{
Tjitske Heida*, Wim L. C. Rutten, and Enrico Marani
}

\begin{abstract}
Recording and stimulating neuronal activity at multiple sites can be realized with planar microelectrode arrays. Efficient use of such arrays requires each site to be covered by at least one neuron. By application of dielectrophoresis (DEP), neurons can be trapped onto these sites. This study investigates negative dielectrophoretic trapping of fetal cortical rat neurons. A planar quadrupole microelectrode structure was used for the creation of a nonuniform electric field. The field was varied in amplitude (1, 3 , and $5 \mathrm{~V}$ ) and frequency $(10 \mathrm{kHz}-50 \mathrm{MHz})$. Experimental results were compared with a theoretical model to investigate the yield (the number of neurons trapped in the center of the electrode structure) with respect to time, amplitude and frequency of the field. The yield was a function of time $\mathrm{e}^{1 / 3}$ according to theory. However, unlike the model predicted, an amplitude-dependent frequency behavior was present and unexpected peaks occurred in the DEP-spectra above $1 \mathrm{MHz}$. Gain/phase measurements showed a rather unpredictable behavior of the electrode plate above $1 \mathrm{MHz}$, and temperature measurement showed that heating of the medium influenced the trapping effect, especially for larger amplitudes and higher frequencies.
\end{abstract}

Index Terms-Cell containment, Clausius-Mosotti factor, cultured cortical neurons, dielectrophoresis (DEP), microelectrode structure, single-shell model.

\section{INTRODUCTION}

$\mathbf{P}$ LANAR microelectrode arrays can be used to provide an interface between a cultured neuronal network and an external electrical circuit for measurement or stimulation [1], [2]. For both applications, neurons should be positioned on, or in the neighborhood of a recording/stimulation site. Several methods for manipulating the location of cultured cells on a substrate exist. High enough walls on a substrate can prevent cells to move across it [3]. By chemical modification, certain regions of the substrate can be made attractive or repulsive for neurons to adhere to (i.e., neurophilic and neurophobic substrates, respectively) [4].

Another method of trapping cells is the use of dielectrophoretic forces [5]-[11]. Dielectrophoresis (DEP) is the phenomenon described by Pohl [9] as the creation of forces

Manuscript received June 5, 2000; revised May 4, 2001. Astreisk indicates corresponding author.

*T. Heida is with the Institute for BioMedical Technology, Faculty of Electrical Engineering, University of Twente, P.O. Box 217, 7500 AE Enschede, The Netherlands (e-mail: t.heida@el.utwente.nl).

W. L. C. Rutten is with the Institute for BioMedical Technology, Faculty of Electrical Engineering, University of Twente, 7500 AE Enschede, The Netherlands.

E. Marani is with the Institute for BioMedical Technology, Faculty of Electrical Engineering, University of Twente, 7500 AE Enschede, The Netherlands, and also with the Department of Neurosurgery, Faculty of Medicine, University of Leiden, 2300 RC Leiden, The Netherlands.

Publisher Item Identifier S 0018-9294(01)06159-6. on neutral, but polarizable particles when they are exposed to nonuniform electric fields. These DEP forces can direct particles toward high field intensities [positive DEP (PDEP)] or toward regions of minimal field intensity [negative DEP (NDEP)]. Next to the (frequency-dependent) electrical properties of particle and medium the DEP force is determined by the particle dimensions and the gradient of the electric field. Field strengths between two and several hundred $\mathrm{kV} / \mathrm{m}$ are required for trapping particles [12].

Experiments showed that several types of living cells were capable to survive the rather high electric fields over longer periods of time up to two days. This was shown for red blood cells, mouse fibroblasts (3T3, L929), suspensor protoplast, bacteria, and yeast [5], [7], [9], [13]. However, high temperatures, which can be caused by an electric field in a medium of high conductivity, can be disastrous [13], [14].

Previous research in the field of DEP has already shown that small particles and living cells can be manipulated by DEP [5], [7], [8], [15]. However, it has thus far not been shown whether this method can be applied to trap neurons. The goal of this paper is to show that dissociated fetal cortical rat neurons can be trapped by negative dielectrophoretic forces. This process was studied qualitatively and quantitatively. A planar quadrupole electrode structure was used for the experiments. The number of neurons trapped in the center of this structure, the yield, was determined for different field settings (amplitudes of 1, 3, and $5 \mathrm{~V}$ and frequencies ranging from $10 \mathrm{kHz}$ to $50 \mathrm{MHz}$ ). A theoretical model was used to determine the relationship of the yield with time, amplitude and frequency of the field. Comparison of this model with the experimental data required investigation of additional aspects like the measurement of the temperature rise of the medium due to the electric field and the signal transfer of the electric circuit. The way of trapping, e.g., the compactness of the group of trapped neurons, was qualitatively determined. From these results an understanding of the dielectrophoretic trapping process was obtained that may lead to accurate trapping of neurons on a recording/stimulation site for a more efficient use of planar multielectrode arrays.

\section{MATERIALS AND MethodS}

\section{A. A Planar Quadrupole Microelectrode Structure}

Fig. 1 schematically shows the electrode structure. The inter-electrode distance between two diagonally opposing electrodes was $100 \mu \mathrm{m}$. A glass substrate was used because of its transparency and thermal conducting properties. On the substrate of $5 \times 5 \mathrm{~cm}$ (thickness $1 \mathrm{~mm}$ ) titanium was applied to improve the adherence of a gold layer onto the glass. Gold 


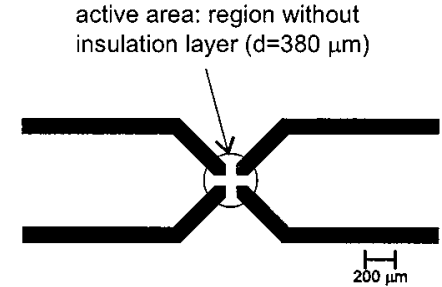

Fig. 1. Schematic drawing of the quadrupole electrode structure.

electrode structures were created by photolithography and reactive ion etching. An insulation layer consisting of a silicone nitride layer $\left(\mathrm{Si}_{x} \mathrm{~N}_{y}\right)$ of $396 \mathrm{~nm}$ sandwiched between two layers of silicon oxide $\left(\mathrm{SiO}_{2}\right)$ of $144 \mathrm{~nm}$ each was deposited by plasma-enhanced chemical vapor deposition. This insulation layer was etched away at the "active regions" and at the connection sites located at one of the edges of the glass plate. After fabrication the electrode plates were cleaned in an ultrasonic bath with acetone. Short thin wires were soldered onto the connection sites and connected with a Bayonet-Neill-Concelman connector that was mounted directly onto the glass plate. A $50-\Omega$ resistor was connected between the two poles of the connector for impedance matching. For the application of a neuron suspension, a culture chamber (a glass ring with an inner diameter of $4 \mathrm{~mm}$ centered round the active region) was placed onto the electrode plate.

\section{B. Electric Field Generation}

For the generation of the electric field in the kHz-range and $\mathrm{MHz}$-range, the following signal generators were used, respectively. HP 3310 A signal generator: Frequency range 0.0001 Hz-5 MHz; Amplitude range 1-16 V. Tektronix Type 191 signal generator: Frequency range $350 \mathrm{kHz}-100 \mathrm{MHz} \pm 2 \%$; Amplitude range $5 \mathrm{mV}-5.5 \mathrm{~V} \pm 5 \%$.

\section{Cells and Medium}

Cortical neurons from rat fetuses (of embryonic day 18, E18) were used for the experiments in this study. The brains were taken out after decapitation, the meninges of the cortices were removed and the basal ganglia as well as the hippocampus were prepared free. The remaining cortices were collected in a tube filled with chemically defined R12 culture medium [16], [17]. The cortices were mechanically dissociated. After precipitating undissociated parts the upper layer of the suspension was removed and centrifuged at a rate of $1200 \mathrm{rpm}$ for $5 \mathrm{~min}$. The medium above the centrifuged neurons was removed and the neurons left at the bottom were again mechanically dissociated using a drop of fresh medium. The last two steps were repeated once. A dilution of $10^{6}$ neurons $/ \mathrm{ml}$ was used.

\section{Measurements}

Using a four point ac-method the conductivity of the R12 medium was measured to be nearly constant $(1.6 \mathrm{~S} / \mathrm{m})$ for frequencies up to $10 \mathrm{kHz}$. Measurement of the viscosity of the medium using a "Contraves low shear" measurement system showed that the medium behaved like a Newtonic fluid $\left(\eta_{\text {med }} \approx 1 \times 10^{-3} \mathrm{~Pa} \cdot \mathrm{s}\right)$.

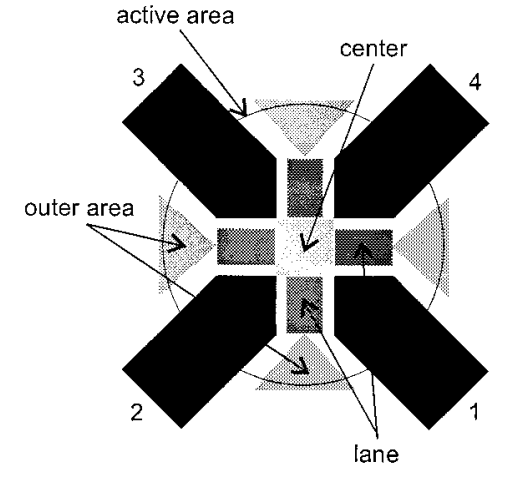

Fig. 2. The electrode structure in detail with the definition of the areas: The center is the square area that is formed by the four electrode tips (center $=5$ $\times 10^{-9} \mathrm{~m}^{2}$ ); the yield is the number of neurons trapped in this square. The lanes are the areas between the parallel sides of the electrode tips leading to the center. The outer areas are triangular areas formed by the diverging electrodes. The electrodes are numbered 1-4.

Temperature measurements were performed using the sensor of an ASEA Fiber thermometer 1010, an optical fiber with a diameter of $100 \mu \mathrm{m}$ positioned above the center of the electrode structure. Medium ( $20 \mu \mathrm{l}$ at room temperature) was pipetted into the culture chamber over which a drop of oil (Klearol-Witco B.V., Koog a/d/Zaan, The Netherlands) was applied to prevent evaporation. The measurement was performed twice for all frequencies.

Gain/phase measurements were performed with a Hewlett Packard HP 4194A Impedance/Gain-Phase analyzer with the following specifications:

$$
\begin{array}{ll}
\text { Frequency range: } & \begin{array}{l}
10 \mathrm{~Hz}-100 \mathrm{MHz} \\
(1-\mathrm{mHz} \text { resolution })
\end{array} \\
\text { Measurement range: } & -107 \mathrm{dBm} \text { to }+15 \mathrm{dBm} \\
& \pm 0.1 \mathrm{~dB}, \pm 180^{\circ} \pm 0.5^{\circ} .
\end{array}
$$

For the investigation of the signal transfer of the electrode structure, a sinusoidal signal generated by the HP analyzer was applied by a probe directly placed onto the connection site of electrode 1 (see Fig. 2). For two different amplitudes (0.5 and $1.2 \mathrm{~V}$ ), the gain and phase were measured by putting a probe (Picoprobe Model 12C - a high-speed, high-impedance, active probe) at the connection sites of the remaining electrodes, one by one, with and without medium applied to the culture chamber. Frequencies up to $60 \mathrm{MHz}$ were used.

\section{E. Experimental Procedure}

The electrode plate was placed in a microscope set up (Nikon Inverted microscope, Diaphot-TMD). A neuron suspension (20 $\mu l)$ was pipetted into the culture chamber while the electric field was applied. Three different amplitudes of the sinusoidal signal from the generator $(1,3$, and $5 \mathrm{~V})$ in combination with ten different frequencies $(10 \mathrm{kHz}, 100 \mathrm{MHz}, 1 \mathrm{MHz}, 4 \mathrm{MHz}, 8 \mathrm{MHz}$, $12 \mathrm{MHz}, 14 \mathrm{MHz}, 18 \mathrm{MHz}, 30 \mathrm{MHz}$, and $50 \mathrm{MHz}$ ) were used. With "amplitude" is meant the amplitude at the output of the signal generator. At the electrode tips, this was not the actual amplitude, but ideally the peak-to-peak value. By a digital video camera (Sony CCD Camera, Model DXC-151P), the experiments were time lapse recorded. Every $10 \mathrm{~s}$, an image was taken during $30 \mathrm{~min}$. Recording started directly after the application of 
the neuron suspension. For each setting of the amplitude and frequency, three experiments were performed. Additionally, three experiments in which no field was applied were performed (reference experiments).

The "yield" is defined as the number of neurons located in the center of the electrode structure during field application. Neurons that did not stay in the center till the end of the experiment were not included in the yield. It was qualified how dense the neurons were packed in the center and the neuron density in the other areas inside the active region as indicated in Fig. 2 was investigated.

\section{THEORY}

\section{A. $D E P$}

The time-averaged dielectrophoretic force $F_{\mathrm{DEP}}$ exerted on a particle $p$ suspended in a medium $m$ exposed to an ac electric field is dependent on the in-phase component of the dipole moment, and can be written as

$$
\begin{aligned}
& \vec{F}_{\mathrm{DEP}}(\omega, x, y, z) \\
& \quad=2 \pi r^{3} \varepsilon_{m} \operatorname{Re}\left[\frac{\varepsilon_{p}^{*}(\omega)-\varepsilon_{m}^{*}(\omega)}{\varepsilon_{p}^{*}(\omega)+2 \varepsilon_{m}^{*}(\omega)}\right] \nabla \vec{E}_{\mathrm{rms}}^{2}(x, y, z)
\end{aligned}
$$

where

index rms root-mean-square value of the electric field;

$\omega=2 \pi f$ with $f$ frequency of the electric field;

$r \quad$ radius of the particle;

$\varepsilon^{*} \quad$ complex permittivity $\varepsilon^{*}(\omega)=\varepsilon-j(\sigma / \omega)$, where $j=(-1)^{1 / 2}$;

$\varepsilon$ and $\sigma \quad$ permittivity and conductivity, respectively.

According to (1) the DEP force is a function of the dimension of the particle, the electrical properties of the particle and the medium, and the distribution of the electric field. The electrical properties of particle and medium are included in the so-called Clausius-Mosotti factor $\left(f_{\mathrm{CM}}\right)$

$$
f_{\mathrm{CM}}(\omega)=\frac{\varepsilon_{p}^{*}(\omega)-\varepsilon_{m}^{*}(\omega)}{\varepsilon_{p}^{*}(\omega)+2 \varepsilon_{m}^{*}(\omega)}
$$

The real part of this factor gives the frequency dependence and the direction of the DEP force (1). When the conductivity and/or permittivity of the medium, each dominating in the $\mathrm{kHz}$ and MHz-range respectively, exceeds that of the cell interior, negative DEP forces will occur. The particles are then directed away from regions of high field intensities. Otherwise, positive forces lead to attraction.

\section{B. Modeling the Electrical Properties of a Suspended Biological Cell}

The active basic molecular mechanisms responding to an external field can be described as polarization and conduction [9]. These responses are frequency-dependent and three dispersions can be distinguished in the range up to $10 \mathrm{GHz}$, i.e., $\alpha, \beta$, and $\gamma$ dispersion each depending on different relaxation mechanisms [15], [18], [19].

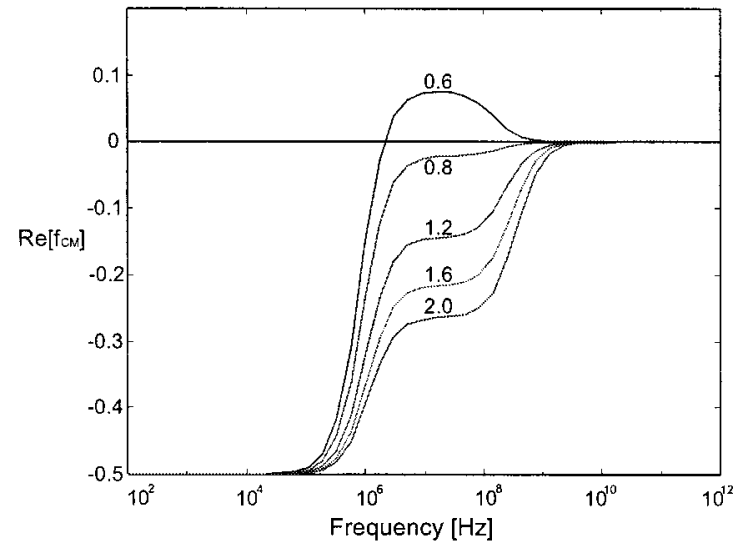

Fig. 3. The real part of the Clausius-Mosotti factor as a function of frequency for a neuron using a single-shell model. Each curve was calculated for a different medium conductivity: $\sigma_{\text {mod }}=0.6,0.8,1.2,1.6$, and $2.0 \mathrm{~S} / \mathrm{m}$.

A $\beta$ dispersion is generally assigned to the Maxwell-Wagner interfacial polarization mechanism, which is considered as the main component responsible for dispersions in biological cells. Interfacial polarization is a consequence of different dielectric constants and conductivities of two media (e.g., particle and suspending medium). It is a "bulk" process, although it produces polarization charges at the interfaces. It appears in the range of $1 \mathrm{kHz}$ to $100 \mathrm{MHz}$ for cell suspensions and biological tissues.

In principle, a cell can be seen as consisting of an interior composed of salty polar fluidic material, around which a membrane with a thickness in the order of $100 \AA$ is present [9]. The membrane has a lipid layer bounded on both sides by polar material, which acts like a capacitor. The capacitance of a membrane is about $1 \mathrm{mF} / \mathrm{m}^{2}$ and its conductance ranges from $10^{-7}$ to $10^{-4} \mathrm{~S} / \mathrm{m}$ [9], [15]. This capacitance can be considered constant since the effect of the relaxation for lipids and proteins is negligible [9].

Water or ionic solutions have a relaxation frequency above $10^{10} \mathrm{~Hz}$. This means that up to this relaxation frequency the permittivity and conductivity of the interior can be considered real and frequency independent.

A model that represents the cell as a homogeneous interior around which a homogeneous membrane is present is the singleshell model [9], [18]. The single-shell model can give rise to one (intrinsic Maxwell-Wagner) dispersion. For a compound dielectric, an "effective pair" of polarization and conduction terms can be determined, which is the effective complex permittivity. A fundamental equation defining the dielectric properties of heterogeneous systems in general can be used to describe the effective complex permittivity for the single-shell model in terms of the permittivities and conductivities of the two regions. Since the thickness of the membrane $\delta$ is small compared to the radius $r$ of the cell $(\delta / r \ll 1)$, this equation can be written as [18], [20]

$$
\varepsilon_{s s, \text { eff }}^{*} \approx c_{\mathrm{mem}} r\left[\frac{j \omega \tau_{\mathrm{int}}+1}{j \omega\left(\tau_{\mathrm{mem}}+\tau_{\mathrm{int}}\right)+1}\right]
$$

where

$$
\begin{aligned}
& \tau_{\text {int }}=\varepsilon_{\text {int }} / \sigma_{\text {int }} \text { and } \\
& \tau_{\text {mem }}=\varepsilon_{\text {mem }} r / \sigma_{\text {int }}
\end{aligned}
$$




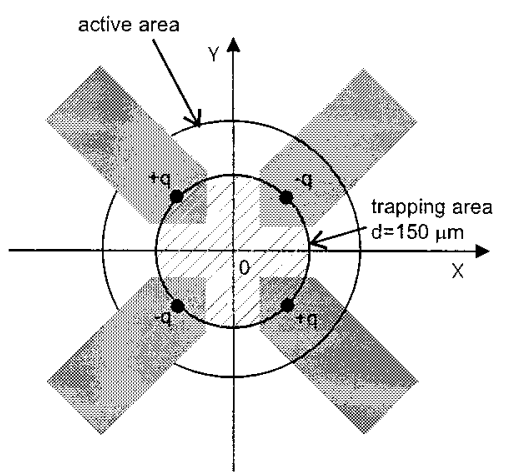

A

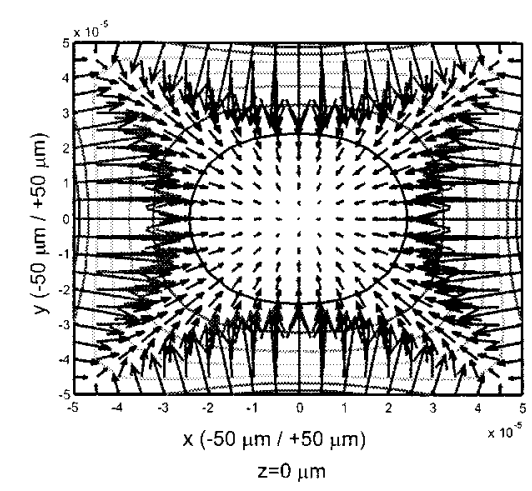

C

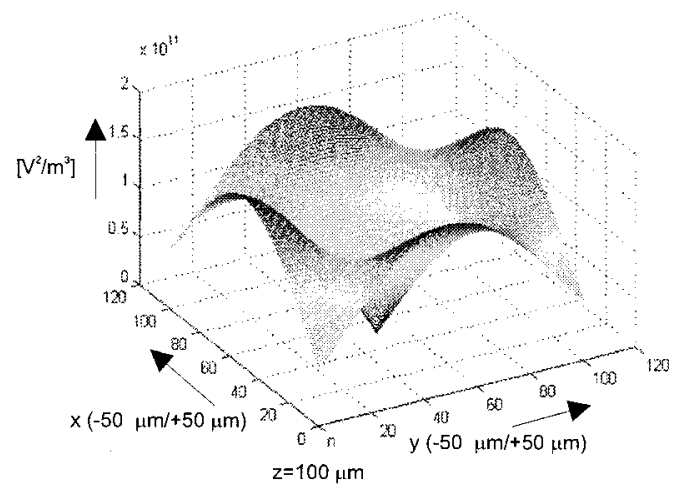

B

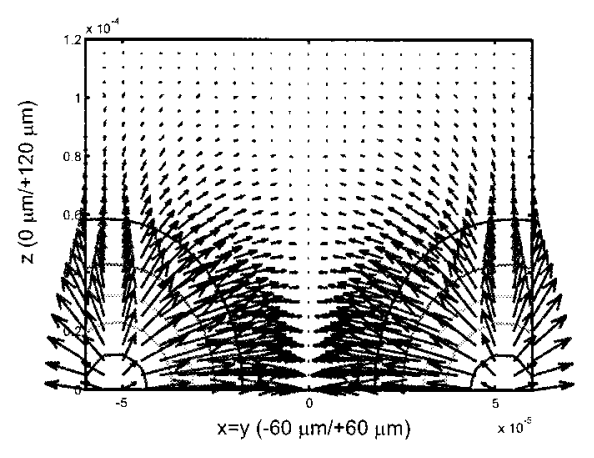

D

Fig. 4. (A) The electrode structure represented by four point charges. The point charges are located at $100 \mu \mathrm{m}$ from the center and are given such a value that the potential at a distance $R=50 \mu \mathrm{m}$ (a distance equal to the "diameter" of the electrode tips) from these points is $5 \mathrm{~V}$ (or $-5 \mathrm{~V}$ ). (B) The relative dielectrophoretic force in the $x y$ plane at $z=100 \mu \mathrm{m}$. The square of the electric field (the solid lines, contours, give the iso-electric field lines) and the gradient of this field (arrows) in (C) the $x y$ plane at $z=0 \mu \mathrm{m}$, and (D) the $(x=y) z$ plane at $z=0 \mu \mathrm{m}$.

$\begin{array}{ll}c \text { mem } & \text { membrane capacitance per unit area, } \\ & c_{\text {mem }}=\varepsilon_{\text {mem }} / \delta, \text { with the index } s s \\ & \text { denoting the single-shell model; } \\ \text { "int" } & \text { inner sphere; } \\ \text { "mem" } & \text { membrane. }\end{array}$

For a suspended cell, a third region is present, which is the suspending medium. By the same argument as that for the cell interior, the permittivity and conductivity of the suspending medium, which is essentially a diluted solution of salt ions in water, can be considered real. The effective complex permittivity of both medium and cell determine the Clausius-Mosotti factor. In effect, this factor may be written as

$$
f_{\mathrm{CM}}=f_{\mathrm{CM}, \infty}+\sum_{k=1}^{2} \frac{\Delta f_{\mathrm{CM}, k}}{1+j \omega v_{k}}
$$

with $\Delta f_{\mathrm{CM}, k}=2 \pi \varepsilon_{m} r^{3}\left|\nabla E_{0, \mathrm{RMS}}^{2}\right| \Delta f_{k}(k=1,2)$, the dispersions in the Clausius-Mosotti factor with relaxation times $\nu_{1}$ and $\nu_{2}, f_{\mathrm{CM}, \infty}$ is the limiting high-frequency value of $f_{\mathrm{CM}}$. The relaxation times correspond to frequencies coinciding with the maximum rate of change of the DEP force.

\section{Clausius-Mosotti Factor for a Neuronal Single-Shell Model}

The following values were assumed (or measured) for the single-shell model of a cortical rat neuron [9], [18]: $r=5 \mu \mathrm{m} ; \sigma_{\text {int }}=0.75 \mathrm{~S} / \mathrm{m} ; \varepsilon_{\mathrm{int}}=\varepsilon_{\mathrm{med}}=80 \times$ Ivarepsilon_ $\{0\}\left(\varepsilon_{0}=8.85 \times 10^{-12} \mathrm{~F} / \mathrm{m}\right) ; c_{\mathrm{mem}}=18 \times 10^{-3} \mathrm{~F} / \mathrm{m}^{2}$ $\left(c_{\mathrm{mem}}=\varepsilon_{\mathrm{mem}} / \delta\right) ; \sigma_{\mathrm{med}}=1.6 \mathrm{~S} / \mathrm{m}$. The graph of Fig. 3 gives the real component of the Clausius-Mosotti factor as a function of frequency for different medium conductivities using (2) and (3). Negative DEP will occur over the entire frequency range up to very high frequencies when the conductivity of the medium is $1 \mathrm{~S} / \mathrm{m}$ or more as was already found [5], [7]. This has the advantage that the cells are distanced from (damaging) high field strengths, and a stable trapping position is created. Small changes in the values for conductivity and permittivity of the interior, the radius of the cell or membrane capacitance did not result in significant changes of this curve (results not shown).

\section{Analytical Analysis of the Electric Field}

To obtain an impression of the distribution of the electric field the quadrupole electrode structure can be represented by four equally valued point charges as shown in Fig. 4(A). From the potential distribution the electric field can be derived and by taking the gradient of the square of this field the relative strength of the DEP force is obtained. Fig. 4(B) shows the funnel created by the electric field and Fig. 4(C) and (D) show the gradient of the squared field for a horizontal and vertical plane, respectively. From these figures it can be concluded that the DEP force is directed toward the center of the four point charges, and it is directed upwards above the electrodes. Only within the area enclosed by the electrodes the DEP force is directed toward the center so that the trapping effect is restricted to this area (trapping area). 


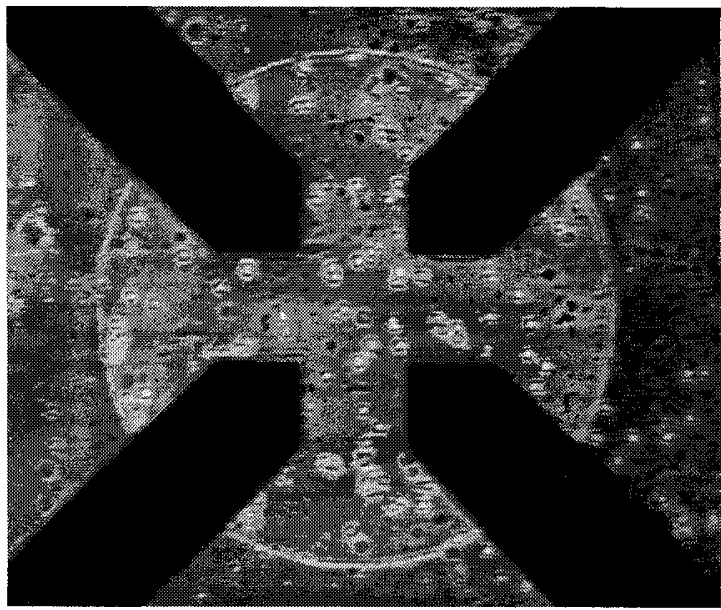

A

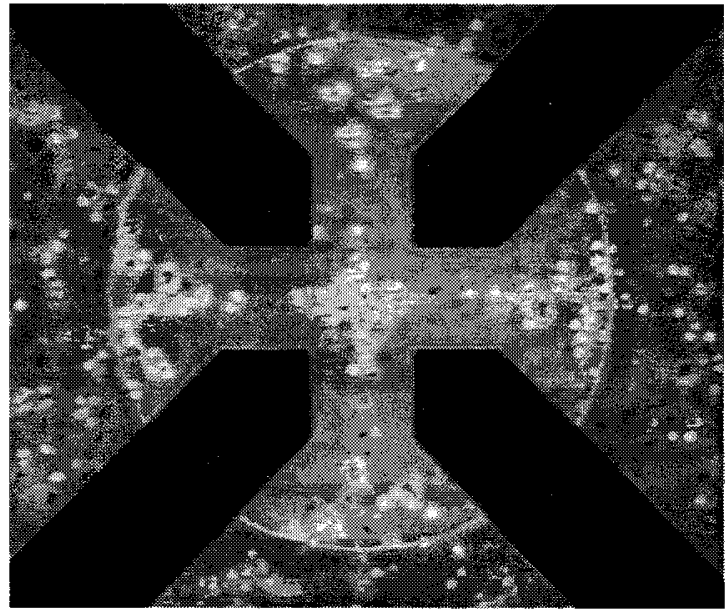

C

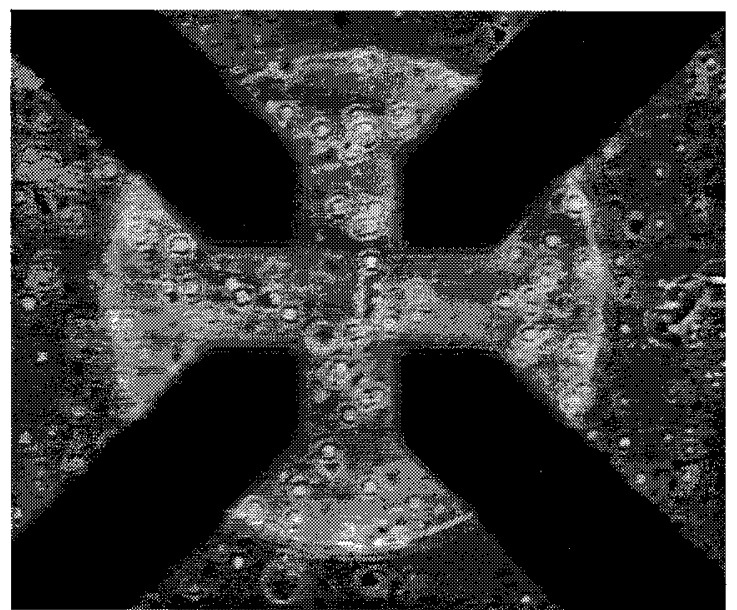

B

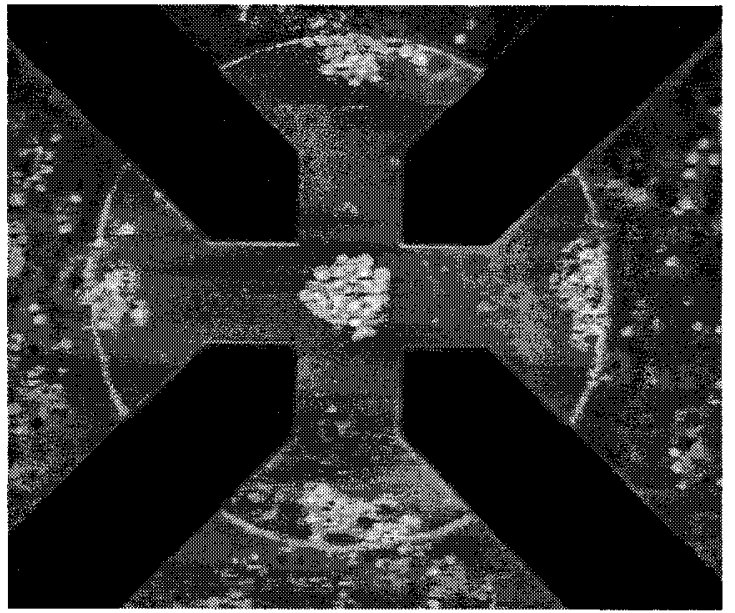

D

Fig. 5. Image nr. 180 of several experiments using different amplitudes (i.e., the situation after 30 min of field application). (A) No input signal (reference experiment), (B) $1 \mathrm{~V} / 12 \mathrm{MHz}$, (C) $3 \mathrm{~V} / 12 \mathrm{MHz}$, and (D) $5 \mathrm{~V} / 12 \mathrm{MHz}$.

\section{E. Theoretical Yield-Force Relationship}

The total force on any particle suspended in a medium of certain conductivity is given by the sum of many forces including Brownian, dielectrophoretic, thermal, hydrodynamic, and sedimentation forces [12], [21]. In the following analysis, it is assumed that only the DEP and drag forces play a significant role.

If the yield is assumed to be a measure of the DEP effect of the electrode structure, a relation to amplitude and frequency of the field should be present. Here, the situation in the $x y$ plane is considered just above the substrate. For the yield, the following relationship was found:

$$
y_{\text {cyl }}=h_{\text {column }} c C_{\text {res }}\left(t q^{2} \operatorname{Re}\left[f_{\mathrm{CM}}\right]\right)^{1 / 3}
$$

with $C_{\text {res }}$ a resulting constant. A detailed derivation of this relationship is given in the Appendix. It is, thus, predicted that the yield is linear with time ${ }^{1 / 3}, \operatorname{Re}\left[f_{\mathrm{CM}}\right]^{1 / 3}$, amplitude ${ }^{2 / 3}$ (amplitude $\sim q$ ). The yield is determined by the neuron concentration $c$ and the height of the column of suspension above the trapping area $h_{\text {column }}$.

\section{RESUlTS}

\section{A. Trapping Neurons under Various Field Conditions}

Fig. 5 shows the localization of trapped neurons after $30 \mathrm{~min}$ under four different field conditions. Image (A) gives the situation in which no field was applied. The other images [(B)-(D)] show situations for three different amplitudes at $12 \mathrm{MHz}$.

In the absence of a field, the neurons precipitated uniformly onto the surface. At $1 \mathrm{~V}$, a uniform positioning of neurons was also obtained [image (B)]. Neurons were trapped in the center of the electrode structure when the amplitude was increased to $3 \mathrm{~V}$ [image (C)]. In the lanes (indicated in Fig. 2), some neurons were also trapped at $3 \mathrm{~V}$. In the outer areas, neurons collected in a triangular shape according to the shape of the diverging electrodes. Increasing the amplitude to $5 \mathrm{~V}$ [image (D)] resulted in a distinct group of neurons collected in the center. The lanes were kept clear of neurons and the distance of neurons to the electrodes in the outer regions was clearly larger than at $3 \mathrm{~V}$.

\section{B. The Yield}

From the reference experiment it was deduced that it took about 10 min for all neurons to precipitate. In this case, an av- 


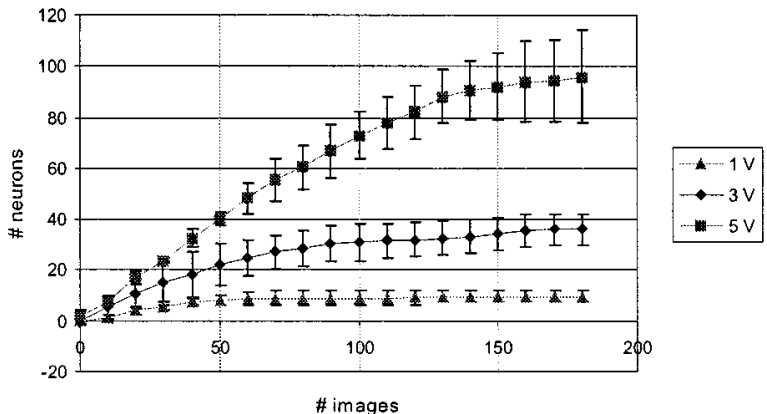

Fig. 6. The average yield of neurons as a function of time ( 10 images $=100 \mathrm{~s})$ at a frequency of $14 \mathrm{MHz}$ for 1,3 , and $5 \mathrm{~V}$. The averages and standard deviations were calculated from the results of three experiments.

TABLE I

THE MINIMUM AND MAXIMUM YIELD FOR 3 AND 5 V

\begin{tabular}{lcccc}
\hline & \multicolumn{2}{c}{ Minimum yield } & \multicolumn{2}{c}{ Maximum yield } \\
\hline amplitude & frequency & \# neurons & frequency & \# neurons \\
$3 \mathrm{~V}$ & $10 \mathrm{kHz}$ & 16 & $1 \mathrm{MHz}$ & 63 \\
$5 \mathrm{~V}$ & $4 \mathrm{MHz}$ & 36 & $14 \mathrm{MHz}$ & 114 \\
\hline
\end{tabular}

erage of nine neurons was located in the center. The $1-\mathrm{V}$ experiments showed a similar result for most frequencies. Fig. 6 gives the average yield as a function of time at $14 \mathrm{MHz}$ for 1 , 3 , and $5 \mathrm{~V}$. Each data point represents the average accumulated number of neurons trapped in the center over 100 s (i.e., ten images). The minimum and maximum yield for 3 and $5 \mathrm{~V}$ is given in Table I. For almost all experiments, the increase in yield was nearly zero during the last minutes (most of the neurons were trapped during the first $20 \mathrm{~min}$ ) of the experiment, indicating a saturation effect. The variation in yield for each field setting was quite large. After $30 \mathrm{~min}$ of field application the average standard deviation was $26 \%$ for $5 \mathrm{~V}$ and $16 \%$ for $3 \mathrm{~V}$.

Fig. 7 shows the yield as a function of frequency (the DEPspectrum) after 30 min of field application. For all amplitudes, a global peak was seen over the first part of the frequency range $(10 \mathrm{kHz}-4 \mathrm{MHz})$. The maximum was located at $1 \mathrm{MHz}$ for 1 and $3 \mathrm{~V}$, and at $100 \mathrm{kHz}$ for $5 \mathrm{~V}$. For frequencies in the range from $4-50 \mathrm{MHz}$, there was again a global peak in the $3-\mathrm{V}$ curve. The maximum was located at $18 \mathrm{MHz}$. Over this frequency range, several peaks were present in the 5-V curve.

\section{Qualitative Aspects of Neuron Trapping}

As could be seen from the image shown in Fig. 5(C) the neurons could be packed quite closely together in the center. The possibility that neurons could be located in the lanes depended on the field parameters [compare image Fig. 5(C) and (D)]. The degree of compactness in the center and the neuron density in the lanes were qualified according to Table II (the 1-V experiments were left out).

\section{Temperature Rise of the Medium Due to the Application of an Electric Field}

Heating of the medium above the center of the electrode structure due to the electric field was found to be amplitude and fre-

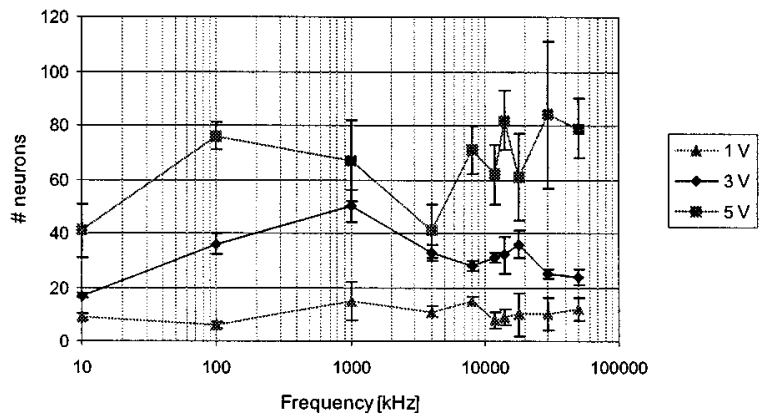

Fig. 7. The yield as a function of frequency after $30 \mathrm{~min}$ of field application.

TABLE II

Compactness of Neuron Clustering in the CENTER AND tHe Neuron DENSITY IN THE LANES

\begin{tabular}{ccccc}
\hline & \multicolumn{3}{c}{$3 \mathrm{~V}$} & \multicolumn{3}{c}{$5 \mathrm{~V}$} \\
\hline Frequency & $\alpha$ & $\beta$ & $\alpha$ & $\beta$ \\
$10 \mathrm{kHz}$ & - & + & 0 & 0 \\
$100 \mathrm{kHz}$ & 0 & 0 & + & 0 \\
$1 \mathrm{MHz}$ & 0 & 0 & + & - \\
$4 \mathrm{MHz}$ & 0 & 0 & + & - \\
$8 \mathrm{MHz}$ & 0 & 0 & + & - \\
$12 \mathrm{MHz}$ & 0 & 0 & + & - \\
$14 \mathrm{MHz}$ & 0 & + & + & - \\
$18 \mathrm{MHz}$ & 0 & 0 & + & - \\
$30 \mathrm{MHz}$ & - & + & 0 & - \\
$50 \mathrm{MHz}$ & - & + & + & - \\
\hline
\end{tabular}

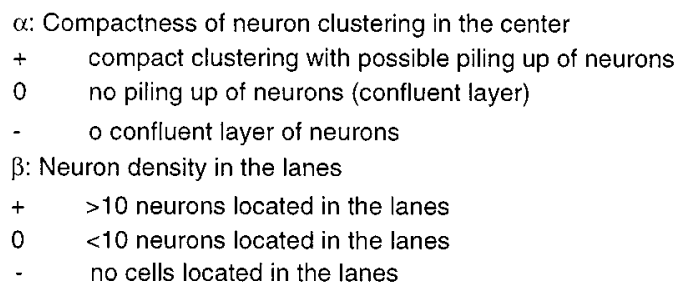

quency dependent. At $1 \mathrm{~V}$, no temperature rise occurred at all. At 3 and $5 \mathrm{~V}$, a temperature rise was noted during the first $5 \mathrm{~min}$ of field application. Thereafter it was stable irrespective the duration of field application. At $5 \mathrm{~V} / 10 \mathrm{kHz}$, the temperature rise was $0.8^{\circ} \mathrm{C}$. With increasing frequency the temperature rise increased. The maximum temperature rise was $1.6^{\circ} \mathrm{C}$ at $5 \mathrm{~V} / 50$ $\mathrm{MHz}$. At $3 \mathrm{~V}$, the maximum temperature rise (at $50 \mathrm{MHz}$ ) was $0.6^{\circ} \mathrm{C}$. It was found that the light from the microscope did not have any effect on the temperature.

\section{E. Signal Transfer of the Electrical Circuit}

Probing three electrodes one by one at their connection sites while the remaining electrode was driven with a sinusoidal input signal showed a nearly constant gain $(\approx 1$ or $0 \mathrm{~dB})$ and phase $\left(\approx 0^{\circ}\right)$ up to about $1 \mathrm{MHz}$ when medium was applied. Above $1 \mathrm{MHz}$, however, deviations from this constant gain and phase 

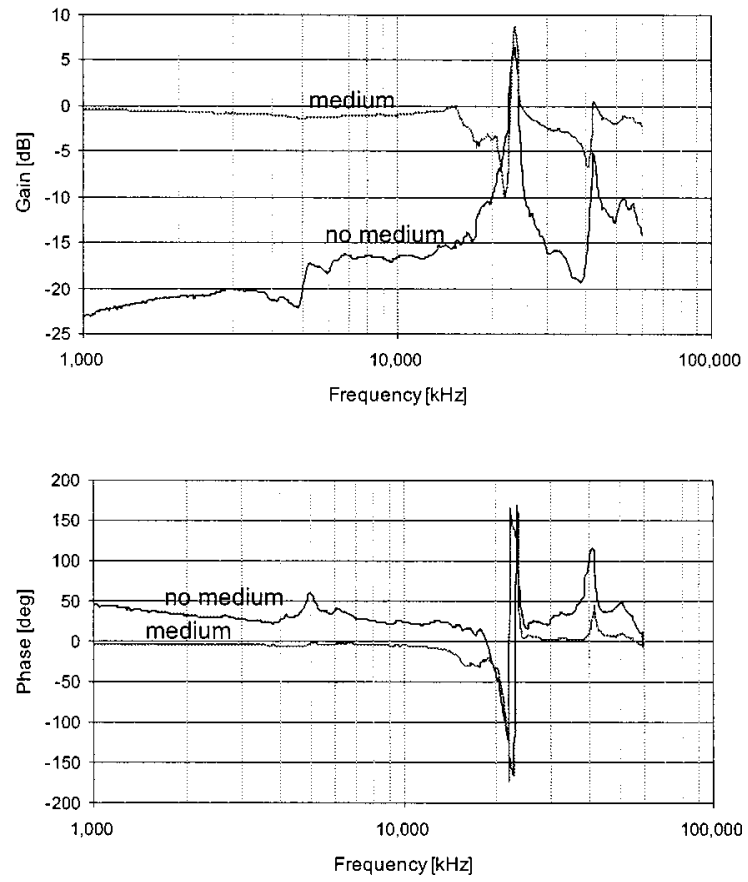

Fig. 8. (a) The average gain and (b) phase of electrodes 2, 3, and 4 when no medium was applied and when medium was applied. The signal amplitude was $1.2 \mathrm{~V}$.

occurred as shown in Fig. 8. The frequency ranged from $1 \mathrm{MHz}$ to $60 \mathrm{MHz}$ and two amplitudes were used $(0.5$ and $1.2 \mathrm{~V})$.

When no medium was applied the gain was much less than one as expected since no conductive medium was present between the electrodes. However, with increasing frequency the gain increased and peaks occurred above $10 \mathrm{MHz}$, comparable to those found when medium was applied. For both amplitudes, the values of the gain did not vary much for the three electrodes $(2,3$, and 4 , see Fig. 2) and no differences in the frequencies at which peaks occurred were found. Fig. 8 shows the average gain and phase for $1.2 \mathrm{~V}$.

\section{DISCUSSION}

\section{A. Qualitative Aspects of Neuron Trapping}

Compact clustering of neurons in the center of the electrode structure went together with lanes that were kept clear of neurons (Table II). Moreover, compactness of trapped neurons was amplitude dependent: the higher the amplitude the more compact the neurons were clustered. Increasing the amplitude will result in a stronger DEP force. Since it is directed upward and sideward near the electrodes an increase in the force results in a decrease of the "width" of the lanes. Neurons that formerly could precipitate in the lanes are now levitated. If next to the DEP force a fluid flow is present, levitated neurons will be swept away and (partially) directed to the center enhancing the process of piling up in the center and clearing the lanes. A fluid flow arises due to local heating of the medium by the electric field. The temperature rise was measured to be largest at $5 \mathrm{~V}$ and it increased with increasing frequency, whereas the DEP force theoretically decreases with increasing frequency. Therefore, it is hard to distinguish for large amplitudes and high frequencies the DEP and fluid flow effects on the trapping process.

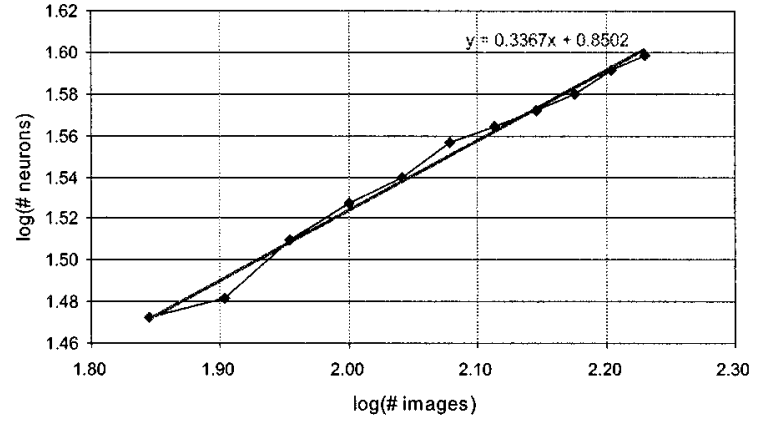

Fig. 9. Verification of the experimental data with the theoretically found relationship between the yield and time. The logarithm of the yield as a function of the logarithm of time for $3 \mathrm{~V} / 18 \mathrm{MHz}$. The slope of the linearly fitted line was 0.3367 .

\section{B. Quantitative Aspects of Neuron Trapping}

In theory, without a field eight neurons would randomly be distributed in the center. The reference experiments showed an average of nine neurons in the center. The creation of a DEP funnel was restricted to the area between the electrode tips. This area was assumed circular with a diameter of $150 \mu \mathrm{m}$ [see, Fig. 4(A)]. About 28 cells would randomly be distributed over this area under zero-field condition. Thus, by using DEP, 28 cells could theoretically at most be trapped in the center.

The results of the $1-\mathrm{V}$ experiments were comparable to the zero-field "yield" and were, therefore, excluded from further analysis. At $3 \mathrm{~V}$, less than 28 cells were trapped at $10 \mathrm{kHz}$ and 30 and $50 \mathrm{MHz}$, after $30 \mathrm{~min}$. In this case, however, more than ten cells were detected in the lanes (Table I). So, in total still about 28 cells were located within the $150-\mu$ m circular area. For an amplitude of $5 \mathrm{~V}$, always more than 28 cells were trapped. This would not be possible if only gravitational and DEP forces were present. From these results it can be deduced that other forces play a role in the trapping effect. As described above, a fluid flow created by heating of the medium may enhance the trapping effect.

Strong variations in the yield occurred between the three experiments for each field setting. In earlier research, large variations were also found. Pohl [9] estimated that a scatter of some $15 \%-20 \%$ in the reproducibility of his yield existed. Differences in cell properties, cell diameter and surface charge density can be ascribed to the large variation in yield [22], [23].

\section{Relationship between the Yield and Experimental Parameters}

Yield-Time Relationship: It was theoretically found that the yield is a function of time ${ }^{1 / 3}$. Fig. 9 shows the logarithm of the curves for $3 \mathrm{~V} / 18 \mathrm{MHz}$. This curve indeed shows a linear behavior with a slope of 0.34. A few data points have been left out of this analysis due to starting and saturation effects. The average slope was 0.347 [standard deviation $(\mathrm{SD})=0.036$ ] and $0.376(\mathrm{SD}=0.096)$ for 3 and $5 \mathrm{~V}$, respectively.

Yield-Amplitude Relationship: For the 3-V experiments, the theoretically assumed cylindrical monolayer of trapped neurons was more likely than for the 5-V experiments (Table II). Therefore, the verification of the yield-amplitude relationship was based on these results. The yield as a function of frequency for 


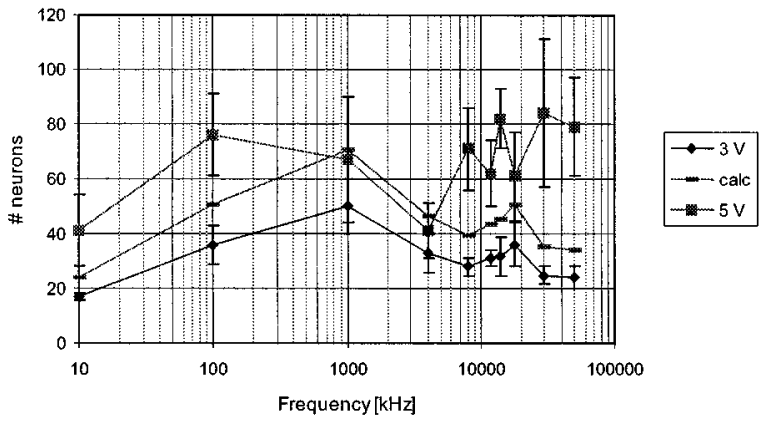

Fig. 10. Verification of the experimental data with the theoretical yieldamplitude relationship. The curves show the yield for 3 and $5 \mathrm{~V}$ after $20 \mathrm{~min}$, and the curve that was calculated using the theoretical yield-amplitude relationship and the experimental $3-\mathrm{V}$ curve.

3 and $5 \mathrm{~V}$ after 20 min (excluding saturation effects) of field application is shown in Fig. 10. Additionally a calculated curve is shown ("calc"). This curve represents the $5-\mathrm{V}$ experiments when the theoretical yield-amplitude relationship is applied to the $3-\mathrm{V}$ results. The curve of the $5-\mathrm{V}$ experiments lies for almost all frequencies above this calculated curve, especially for the higher frequencies as expected, but it seems that an amplitude-dependent frequency behavior was present.

Yield-Re $\operatorname{Re}\left[f_{\mathrm{CM}}\right]$ Relationship: The yield- $\operatorname{Re}\left[f_{\mathrm{CM}}\right]$ relationship in effect gives the yield-frequency relationship. Therefore, both factors (yield and $\operatorname{Re}\left[f_{\mathrm{CM}}\right]$ ) were compared with respect to frequency. In theory, $\operatorname{Re}\left[f_{\mathrm{CM}}\right]$ is negative over the whole frequency range used. Experimentally, the neurons were indeed repelled from the electrodes for all frequencies. Fig. 3 showed that with increasing frequency $\operatorname{Re}\left[f_{\mathrm{CM}}\right]$ decreased. This was not the case for some parts of the yield curves (Fig. 7).

The experimental frequency behavior of trapping not only includes the frequency dependence of the neurons and medium as included in $f_{\mathrm{CM}}$, but also the frequency dependence of the signal transfer properties of the whole system. When the wavelength $\lambda$ of the signal is much larger than the largest dimension of the device or system a low-frequency approximation can be used. Simulating the electrode plate an electric circuit consisting of resistors and capacitors resulted in a low-pass behavior with a cutoff frequency of about $100 \mathrm{MHz}$. However, the gain/phase measurements did show a constant gain and phase up to about 1 $\mathrm{MHz}$; above $1 \mathrm{MHz}$, the gain showed a rather unpredictable behavior. This might explain the occurrence of peaks in the $(5-\mathrm{V})$ DEP results above $1 \mathrm{MHz}$.

As already indicated, the experimental yield-amplitude relationship postulates an amplitude-dependent frequency behavior. This dependence is not present in the Clausius-Mosotti factor, which implies that the medium and/or neuron was not adequately described or that the electrode plate showed an amplitude-dependent frequency behavior. The gain/phase measurements with and without medium did not result in substantial shifts or addition of gain peaks. The medium obviously did not change its properties over the frequency range used. Additionally, the gain/phase measurements did not show an amplitude-dependent behavior over the frequency range used (not shown).
As was already stated in literature [8], a fluid flow created by local heating can enhance the trapping effect in a planar quadrupole electrode structure. Especially at larger amplitudes and higher frequencies heating became more apparent, with the result that it becomes difficult to distinguish whether the force created by the flow or the DEP force caused trapping. For the $5-\mathrm{V}$ results, it can be concluded that the DEP effect was overshadowed by the temperature effect, especially for the higher frequencies. Therefore, the 3-V results are most reliable to compare the experimental frequency behavior with the theoretical yield-frequency relationship (i.e., yield- $\operatorname{Re}\left[f_{\mathrm{CM}}\right]$ relationship). Except for a small peak at $18 \mathrm{MHz}$ the frequency behavior the $3-\mathrm{V}$ results did show similarities with the expected behavior for frequencies above $1 \mathrm{MHz}$. However, the result at 10 and 100 $\mathrm{kHz}$ can not be explained.

\section{Additional Considerations}

The expression for the DEP force (1) is based on the equivalent dipole method, in which the force is calculated as the interaction of the induced dipole moment and the nonuniform external field. The assumptions made in the derivation are [24], [25]: 1) the applied field varies sinusoidally in time; 2) the media are isotropic and linear, i.e., $\varepsilon$ and $\sigma$ are not functions of the field orientation or the field strength; 3 ) the frequency of the field is low enough to permit the electric field to be derived from a scalar potential; 4) the external field is assumed to be uniform in the vicinity of the sphere; and 5) a single spherical particle is considered.

When a particle is located in a strong electric field gradient or near a null field, (1) does not give an adequate description. Higher order multipolar moments must be taken into account in these situations [6], [25]. Another aspect is mutual DEP. Particles positioned in a nonuniform field distort the field making it more nonuniform in their vicinity. Since only one particle was considered for the derivation of the DEP force the effect of mutual DEP was neglected. It has been found that mutual DEP can create a rather enhanced trapping effect [10], [26].

It is possible to discriminate between cells on the basis of their physiological state. For example, DEP-spectra of viable and nonviable yeast cells show substantial differences [23]. Furthermore, differences between normal and cancerous cells [27] have been characterized. Using frequencies below $100 \mathrm{kHz}$ may cause irreversible damage due to loading of the membrane. This might explain the difference between theory and experiment for the low-frequency range. Therefore, the single-shell model as used for the whole frequency range may only be applicable to the upper part of this range.

Since the maximum temperature rise was only $1.6^{\circ} \mathrm{C}$ for the higher frequencies this was not expected to have harmful effects on the viability of the cells.

\section{CONCLUSION}

This paper shows the applicability of DEP for trapping cortical rat neurons. The trapping process depends on the field settings, as well as the electrical properties of neuron and suspending medium. Several aspects have been investigated 
leading to a better understanding of the trapping process. The creation of a fluid flow due to local heating of the medium was ascribed to be the main reason for the discrepancies between theory and experiment, especially for larger amplitudes and higher frequencies. The single-shell model may not represent the neuron properly over the whole frequency range used. Investigating the physiological state of the neuron after trapping at different field settings may result in a more accurate model of the neuron. New designs of electrode structures may result in trapping neurons on multiple sites and additionally in a more efficient use of microelectrode arrays.

\section{APPENDIX}

\section{A. Theoretical Yield-Force Relationship}

Assume the neurons have precipitated onto the surface near the electrode tips of the electrode structure. Here, the negative dielectrophoretic force is directed in a horizontal way and the neuron is translated in a plane perpendicular to the surface. When a spherical particle with radius rmoves in a medium it experiences a viscous drag force (Stokes' law). This force is oppositely directed to the dielectrophoretic force. A force balance consisting of an acceleration term, and a dielectrophoretic and viscous component. Taking the velocity of the cell to be constant a dynamic equilibrium is obtained and, thus, the resultant of the dielectrophoretic and viscous forces is zero

$$
\vec{F}_{\text {acc }}=\vec{F}_{\mathrm{DEP}}+\vec{F}_{\text {viscous }}=0 .
$$

The following equations describe the dielectrophoretic force and the viscous force, respectively:

$$
\begin{aligned}
\vec{F}_{\mathrm{DEP}}(\omega, x, y, z) & =2 \pi r^{3} \varepsilon_{m}^{\prime} \operatorname{Re}\left[f_{\mathrm{CM}}(\omega)\right] \nabla \vec{E}_{\mathrm{rms}}^{2}(x, y, z) \\
\vec{F}_{\text {viscous }}(v) & =-6 \pi \eta r \vec{v} .
\end{aligned}
$$

For a constant frequency of the field, these two equations can be solved for the velocity

$$
v=\frac{1}{3 \eta} r^{2} \varepsilon_{m}^{\prime}, \operatorname{Re}\left[f_{\mathrm{CM}}\right]\left|\nabla \vec{E}_{\mathrm{rms}}^{2}\right|
$$

Assume a neuron travels from $R_{0}$ to a point $R_{c}$ close to the center in a horizontal plane with velocity $v$. Mathematically, the situation is given in Fig. 11. Representing each electrode by a point charge an analytical equation for the electric field was obtained so that the last factor in (A.4) at a distance $R$ from the point charge becomes

$$
\left|\nabla E^{2}\right|=C_{1}^{2} \frac{q^{2}}{R^{5}}
$$

For the velocity, this means

$$
v=C_{2} \operatorname{Re}\left[f_{\mathrm{CM}}\right] \frac{q^{2}}{R^{5}}
$$

where the constant $C_{1}$ and the constant parameters from (A.4) were included into the constant $C_{2}$. The time required for a

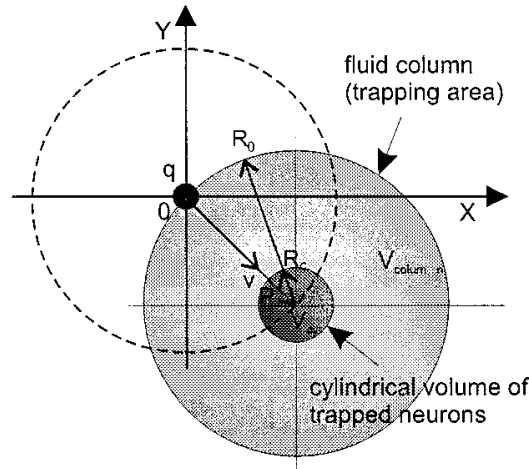

Fig. 11 A neuron travels from an electrode tip toward the center of the quadrupole electrode structure. Mathematical description of the "real" situation.

neuron to travel from $R_{0}$ to a point $R_{c}$ close to the center in a horizontal plane is given by $\left(C_{3}=1 / C_{2}\right)$

$t=\int_{P-R_{0}}^{P-R_{c}} \frac{C_{3} R^{5}}{q^{2} \operatorname{Re}\left[f_{\mathrm{CM}}\right]} d R=\frac{C_{3}\left(\left(P-R_{c}\right)^{6}-\left(P-R_{0}\right)^{6}\right)}{q^{2} \operatorname{Re}\left[f_{\mathrm{CM}}\right]}$.

The neurons collect in the center of the quadrupole electrode structure. The volume of neurons can be represented by a cylinder $V_{\text {cyl }}$ with a height $h$ of $10 \mu \mathrm{m}$ (a monolayer of neurons, $h=2 r$ ). The yield $y_{\text {cyl }}$ is the number of neurons within this cylinder.

If the area with a diameter of $150 \mu \mathrm{m}$ is considered as the trapping area, the column of neuron suspension (the starting volume $V_{\text {column }}$ indicated in Fig. 11) above this area contains all neurons that will be trapped. The volume of trapped neurons then becomes

$$
y_{\mathrm{cyl}}=\frac{V_{\mathrm{cyl}}}{V_{n}}=c V_{\mathrm{column}}=c\left(\pi h_{\mathrm{column}} R_{\text {column }}^{2}\right)
$$

with $c$ the neuron concentration, and $V_{n}$ is the volume of a neuron $\left[V_{n}=(4 / 3) \pi r^{3}\right.$, with $r$ the radius of a neuron $(r \approx 5$ $\mu \mathrm{m})$ ], $h_{\text {column }}$ and $R_{\text {column }}$ are the height and the radius of the suspension column, respectively. The starting volume is swept out in time $t$. Taking $P=R_{0}=R_{\text {column }}$ and $R_{c}=0$, then the expression for the yield can be found by combining (A.7) with (A.8).

$$
y_{\text {cyl }}=h_{\text {column }} c C_{\text {res }}\left(t q^{2} \operatorname{Re}\left[f_{\mathrm{CM}}\right]\right)^{1 / 3}
$$

with $C_{\text {res }}$ a constant resulting from all constant factors.

\section{ACKNOWLEDGMENT}

The authors would like to thank J. van Nieuwkasteele, T. Verloop, and E. Droog for the technical support of the fabrication of the microelectrode plate and M. Deenen for cortex neuron preparation.

\section{REFERENCES}

[1] M. P. Maher, J. Pine, J. Wright, and Y. C. Tai, "The neurochip: A new multielectrode device for stimulating and recording from cultured neurons," J. Neurosci. Meth., vol. 87, no. 1, pp. 45-56, 1999. 
[2] W. G. Regehr, J. Pine, and D. B. Rutledge, "A long-term in vitro siliconbased microelectrode-neuron connection," IEEE Trans. Biomed. Eng., vol. BME-35, pp. 1023-1031, 1988.

[3] C. D. W. Wilkinson, "Nanostructures in biology," Microelectron. Eng., vol. 27, pp. 61-65, 1995 .

[4] D. W. Branch, J. M. Corey, J. A. Weyhenmeyer, G. J. Brewer, and B. C. Wheeler, "Microstamp patterns of biomolecules for high-resolution neuronal networks," Med. Biol. Eng. Comput., vol. 36, pp. 135-141, 1998.

[5] G. Fuhr, T. Müller, T. Schnelle, R. Hagedorn, A. Voigt, S. Fiedler, W. M. Arnold, U. Zimmermann, B. Wagner, and A. Heuberger, "Radio-frequency microtools for particle and live cell manipulation," Naturwissenschaften, vol. 81, pp. 528-535, 1994.

[6] T. B. Jones and M. Washizu, "Multipolar dielectrophoretic and electrorotation theory," J. Electrostat., vol. 37, pp. 121-134, 1996.

[7] G. H. Markx and R. Pethig, "Dielectrophoretic separation of cells: Continuous separation," Biotech. Bioeng., vol. 45, pp. 337-343, 1995.

[8] T. Müller, A. Gerardino, T. Schnelle, S. G. Shirley, F. Bordoni, G. De Gasperis, R. Leoni, and G. Fuhr, "Trapping of micrometer and sub-micrometer particles by high-frequency electric fields and hydrodynamic forces," J. Phys. D: Appl. Phys., vol. 29, pp. 340-349, 1996.

[9] H. A. Pohl, Dielectrophoresis. Cambridge, U.K.: Cambridge University Press, 1978.

[10] T. Schnelle, T. Müller, S. Fiedler, S. G. Shirley, K. Ludwig, A. Herrmann, G. Fuhr, B. Wagner, and U. Zimmermann, "Trapping of viruses in high-frequency electric field cages," Naturwissenschaften, vol. 83, pp. 172-176, 1996.

[11] X.-B. Wang, Y. Huang, R. Hölzel, J. P. H. Burt, and R. Pethig, "Theoretical and experimental investigations of the interdependence of the dielectric, dielectrophoretic and eletrorotational behavior of colloidal particles," J. Phys. D: Appl. Phys., vol. 26, pp. 312-322, 1993.

[12] G. Fuhr and S. G. Shirley, "Cell handling and characterization using micron and submicron electrode arrays: State of the art and perspectives of semiconductor microtools," J. Micromech. Microeng., vol. 5, pp. 77-85, 1995.

[13] J.-Z. Bao, C. C. Davis, and R. E. Schmukler, "Frequency domain impedance measurements of erythrocytes: Constant phase angle impedance characteristics and a phase transition," Biophys. J., vol. 61, pp. 1427-1434, 1992

[14] G. Fuhr, A. Voigt, T. Müller, B. Wagner, K. Reimer, and T. Lisec, "Electric-field-mediated inhibition of cell and microparticle adhesion: A new way to create bio-repellent surfaces," Sensors Actuators B: Chem., vol. 26-27, pp. 468-470, 1995.

[15] R. Pethig, "Application of AC electrical fields to the manipulation and characterization of cells," in Automation in Biotechnology-Proc. 4th Conf., I. Karube, Ed., 1990, pp. 159-185.

[16] E. Marani, M. Corino, R. J. van den Berg, W. J. Rietveld, M. Deenen, and W. Windhorst, "Ionic conductances in cultured pre-infundibular cells from the hypothalamic arcuate region," Neuroendocrinology, vol. 48, pp. $445-452,1988$.

[17] H. J. Romijn, F. van Huizen, and P. S. Wolters, "Toward an improved serum-free, chemically defined medium for long-term culturing of cerebral cortex tissue," Neurosci. Biobehav. Rev., vol. 8, pp. 301-334, 1984.

[18] K. V. I. S. Kaler and T. B. Jones, "Dielectrophoretic spectra of single cells determined by feedback-controlled levitation," Biophys. J., vol. 57, pp. 173-182, 1990 .

[19] J. Gimsa and D. Wachner, "A unified resistor-capacitor model for impedance, dielectrophoresis, electrorotation, and induced transmembrane potential," Biophys. J., vol. 75, pp. 1107-1116, 1998.

[20] A. Irimajiri, "A dielectric theory of 'multi-stratified shell' model with its application to a lymphoma cell," J. Theor. Biol., vol. 78, pp. 251-269, 1979.

[21] A. Ramos, H. Morgan, N. G. Green, and A. Castellanos, "AC elektrokinetics: A review of fores in microelectrode structures," J. Phys. D: Appl. Phys., vol. 31, pp. 2338-2353, 1998.
[22] N. G. Green and H. Morgan, "Dielectrophoretic investigations of sub-micrometer latex spheres," J. Phys. D: Appl. Phys., vol. 30, pp. 2626-2633, 1997.

[23] Y. Huang, R. Hölzel, R. Pethig, and X.-B. Wang, "Differences in the AC electrodynamics of viable and nonviable yeast cells determined through combined dielectrophoresis and electrorotation studies," Phys. Med. Biol., vol. 37, no. 7, pp. 1499-1517, 1992.

[24] X.-B. Wang, Y. Huang, F. F. Becker, and P. R. C. Gascoyne, "A unified theory of dielectrophoresis and travelling wave dielectrophoresis," J. Phys. D: Appl. Phys., vol. 27, pp. 1571-1574, 1994.

[25] M. Washizu, "Precise calculation of dielectrophoretic force in arbitrary field," J. Electrostat., vol. 29, pp. 177-188, 1992.

[26] T. Schnelle, T. Müller, and G. Fuhr, "Dielectric single particle spectroscopy for measurement of dispersion," Med. Biol. Eng. Comput., vol. 37, pp. 264-271, 1999

[27] P. R. C. Gascoyne, J. Noshari, F. F. Becker, and R. Pethig, "Use of dielectrophoretic collection spectra for characterizing differences between normal and cancerous cells," IEEE Trans. Ind. Applicat., vol. 30, pp. 829-834, July/Aug. 1994.

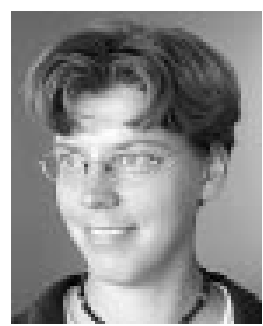

Tjitske Heida received the M.D. degree in electrical engineering from the University of Twente, Twente, The Netherlands, in 1997. Presently, she is working there as a Ph.D. degree student in the Signals and Systems group/BMTI

Her research interests include investigation of the use of dielectropheresis for trapping neural cells on the electrodes of a multielectrode array for stimulation and measurement purposes.

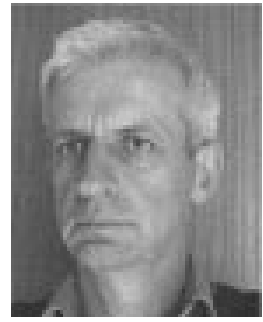

Wim L. C. Rutten was trained as an Experimental Physicist (received the Ph.D. degree in 1979) at Leiden University, Leiden, The Netherlands. Thereafter he studied the auditory system at the ENT department of Leiden University Hospital.

Since 1985, he is an Assistant and Associate Professor of Biomedical Control Systems with BMTI/Faculty of Electrical Engineering, University of Twente, Twente, The Netherlands. His present research interests are neural engineering and cellular engineering (neuro-electronic interfaces, cultured probes), signal processing, and bioelectricity.

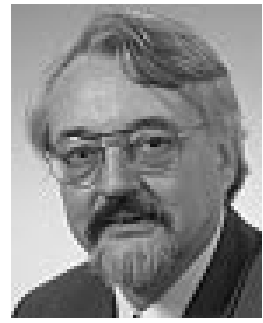

Enrico Marani was trained as a Neuroanatomist (received the Ph.D. degree in 1982) at the Leiden University, Leiden, The Netherlands.

Thereafter, he became Head of the Neuroregulation group at the Department of Neurosurgery, Leiden University Medical Center. Since 1997, he has been a Part-time Professor of Neurophysiology at the BMTI/Faculty of Electrical Engineering of the University of Twente, Twente, The Netherlands. His present research interests are neuroregulation and neurotechnology, especially in the field of nerve 